\title{
Two Alternative Proposals for Abbreviations of Names of Genera Suggested for Coding Microbiological Data
}

\author{
W. LEVERITT AND V. B. D. SKERMAN \\ World Data Center for Microorganisms, Department of Microbiology, University of Queensland, St. Lucia, \\ Queensland, Australia
}

Two alternative proposals for abbreviations of names of genera are suggested for coding microbiological data and are compared to that of Johnson et al. (1976).

The proposal for abbreviations of generic names by Johnson et al. (1) offers a solution to a continual problem facing systems analysts and computer programmers: a type of "inverse Parkinson's Law" problem where "the amount of data must be contracted to fit the available computer space." In this instance the problem is caused by the variable length of names of taxa.

However, the list of abbreviations as proposed by Johnson et al. does provide a difficulty for the computer programmer who wishes to use the computer to perform changes from generic names to abbreviations and vice versa and to automatically arrange the names or abbreviations in alphabetical order for indexing purposes. The problem is that the letters in each abbreviation are not in alphabetic sequence. This is significant for the following reason.

When performing interchanges of this nature by computer it is the normal procedure to arrange the information in a two-column table within the computer memory; in this case generic names are placed in column 1 and abbreviations are placed in column 2 . To find an abbreviation for any generic name, one has simply to search down column 1 for the name, and then obtain the abbreviation from the same row in column 2 . The procedure by which the search is performed is significant. If the generic names are in any order and a sequential searching method is applied (i.e., the names are looked at in turn, starting with the first, and following with the second, third, etc.), then a list of 64 names would require an average of 32 comparisons to find any given name. If, however, the names are in alphabetic order, a procedure such as the binary search in the Appendix can be applied. This procedure would find any name in an ordered list of length of 64 names in no more than 8 comparisons. Such savings in the number of comparisons needed can become extremely important where repeated searches of large lists are required.

The proposed list of Johnson et al. is perfectly satisfactory for conversion from generic name to abbreviation using the binary search technique, as the generic names are in alphabetic order. However, to convert from abreviation to generic name requires either of the following:

(i) a second table, with column 1 being the abbreviations in alphabetic order and column 2 being the generic names.

This second table of approximately 300 genera and abbreviations would require additional computer memory. (On the PDP-10 computer used by the World Data Center for Microorganisms at the University of Queensland, Brisbane, Australia, each table would occupy $5 \%$ of the normally available main memory space).

(ii) a sequential search down column 2 of

the original table, causing an increase in computer time through increased numbers of comparisons.

Both of the above become unnecessary if the list of abbreviations is also compiled in alphabetic sequence to correspond to the list of generic names. With both names and abbreviations in alphabetic sequence (i) only one table is required in the computer memory and a single searching procedure (the binary search) can be used for conversions from generic name to abbreviation and vice versa, and (ii) the abbreviations can be used for indexing.

Such a four-character abbreviation list is specified in Table 1 . In producing this list, an attempt was made to follow the original list and the code of abbreviations of Johnson et al.

Johnson et al. admit their list contains some inconsistencies. Further inconsistencies were, by necessity, introduced to force alphabetic ordering into the four-character abbreviation. Of particular note is the change in the Code for Abbreviations (Table 1 of reference 1)-BACILLUS becomes BA and BACTER etc. becomes BC. Johnson et al. only use letters in the abbreviation which are present in the full generic name. This has not been possible in the alphabetic list; thus, BARTONELLA becomes BDTL, ACTINOPYCNIDIUM becomes ACTQ. Many of the inconsistencies can be removed 
TABLE 1. Abbreviations for bacterial genera

\begin{tabular}{|c|c|c|c|c|c|c|c|}
\hline Genus & $\begin{array}{c}\text { Johnson } \\
\text { et al. } \\
\text { proposal } \\
(1)\end{array}$ & $\begin{array}{c}\text { 4-Char- } \\
\text { acter al- } \\
\text { pha- } \\
\text { betic } \\
\text { pro- } \\
\text { posal }\end{array}$ & $\begin{array}{l}\text { 5-Charac- } \\
\text { ter alpha- } \\
\text { betic pro- } \\
\text { posal }\end{array}$ & Genus & $\begin{array}{l}\text { Johnson } \\
\text { et al. } \\
\text { proposal } \\
(1)\end{array}$ & $\begin{array}{c}\text { 4-Char- } \\
\text { acter al- } \\
\text { pha- } \\
\text { betic } \\
\text { pro- } \\
\text { posal }\end{array}$ & $\begin{array}{c}\text { 5-Charac- } \\
\text { ter alpha- } \\
\text { betic pro- } \\
\text { posal }\end{array}$ \\
\hline Acetobacter & ACBT & ACBC & ACEBC & Cristispira & CRSP & CRSP & CRTSP \\
\hline Acholeplasma & ACPL & ACHL & ACHLP & Cystobacter & CSBT & CSBC & CYSBC \\
\hline Achromatium & ACMT & ACHM & ACHMT & Cytophaga & CTFG & CTFG & CYTFG \\
\hline Achroonema & ACNM & ACHR & ACHRO & Dactylosporangium & DTSO & DCSO & DCTSO \\
\hline Acidaminococcus & AACO & ACIC & ACICO & Dermatophilus & DMFL & DEMF & DEMFL \\
\hline Acinetobacter & ANBT & ACNB & ACNBC & Derxia & DRXA & DERX & DERXA \\
\hline Actinobacillus & ATBC & ACTB & ACTBA & Desmanthos & DMTS & DESM & DESMT \\
\hline Actinobifida & ATBI & ACTF & ACTBI & Desulfomonas & Not listed & DESN & DESNS \\
\hline Actinomyces & ATMY & ACTN & ACTMY & Desulfotomaculum & DSMC & DEST & DESTM \\
\hline Actinoplanes & ATPN & ACTP & ACTPN & Desulfovibrio & DSVB & DESV & DESVB \\
\hline Aegyptianella & AGTL & AEGT & AEGTL & Ectothiorhodospira & ETSP & ECSP & ECTSP \\
\hline Aerococcus & ARCO & AERC & AERCO & Edwardsiella & EDWL & EDWL & EDWSL \\
\hline Aeromonas & ARMN & AERM & AERMN & Ehrlichia & EHRL & EHRL & EHRLC \\
\hline Agrobacterium & AGBT & AGBC & AGRBC & Enterobacter & ENBT & ENBC & ENTBC \\
\hline Alcaligenes & ALC & ALCA & ALCAL & Eperythrozoon & EPRZ & EPRZ & EPRYZ \\
\hline Alysiella & ALSL & ALSL & ALYSL & Erwinia & ERWN & ERWN & ERWNA \\
\hline Amoebobacter & AMBT & AMBC & $\mathrm{AMBBC}$ & Erysipelothrix & ESTX & ERYT & ERYTX \\
\hline Amorphosporangium & AMSP & AMFS & AMFSP & Escherichia & ESCH & $\mathrm{ESCH}$ & ESCHR \\
\hline Ampullariella & APRL & AMPL & AMPRL & Eubacterium & EUBT & EUBC & EUABC \\
\hline Anaerovibrio & Not listed & ANAE & ANAEV & Flavobacterium & FVB'T & FLVB & FLVBC \\
\hline Anaplasma & ANPL & ANAP & ANAPL & Flexibacter & FXBT & FLXB & FLXBC \\
\hline Ancalomicrobium & ANMI & ANMI & ANCMI & Flexithrix & FXTX & FLXT & FLXTX \\
\hline Arachnia & ARAC & ARAC & ARACH & Francisella & FRCL & FRCL & FRNCL \\
\hline Archangium & ACNG & ARCN & ARCNG & Frankia & FRNK & FRNK & FRNKA \\
\hline Arthrobacter & ARBT & ATBC & ARTBC & Fusobacterium & FSBT & FSOB & FSOBC \\
\hline Asticcacaulis & ASCL & ATCL & ASTCL & Gallionella & GLNL & GALN & GALNL \\
\hline Azomonas & AZMN & AZMN & AZOMN & Gemella & GMLL & GEMA & GEMLA \\
\hline Azotobacter & AZBT & AZTB & AZTBC & Geodermatophilus & GDMF & GEMF & GEODF \\
\hline Bacillus & BC & BACL & BACLS & Gluconobacter & GNBT & GNBC & GLNBC \\
\hline Bacterionema & BTNM & BCNM & BAC & Grahamella & GRML & GRML & GRHML \\
\hline Bacteroides & BTRD & BCRD & BACRD & Haemobartonella & HMBL & HAMB & HAMBL \\
\hline Bartonella & BRTL & BDTL & BA & Haemophilus & HMFL & HAMP & HAMPL \\
\hline Bdellovibrio & BDVB & BDVB & BD & Hafnia & HAFN & HFNA & HFNIA \\
\hline Beggiatoa & BGGT & BEGT & BEGIT & Halobacterium & HLBT & HLBC & HLOBC \\
\hline Beijerinckia & BEIJ & BEIJ & BEIJK & Halococcus & HLCO & HLCO & HLOCO \\
\hline Bifidobacterium & BIBT & BIBC & BIFBC & Herpetosiphon & HPSN & HRSN & HRTSN \\
\hline Blastobacter & BSBT & BLSB & BLSBC & Hyphomicrobium & HFMI & HYMI & HYFMI \\
\hline Blattabacterium & BLBT & BLTB & BLTBC & Hyphomonas & HFMN & HYMN & HYFMN \\
\hline Bordetella & BDTL & BORD & BORDL & Kingella & Not listed & KING & KINGL \\
\hline Borrelia & BRRL & BORL & BO & Kitasatoa & KTST & KITS & KITST \\
\hline Brachyarcus & BRAC & BRAC & BR & Klebsiella & KLEB & KLEB & KLEBS \\
\hline Branhamella & BNHL & BRAN & BRANH & Kurthia & KURT & KURT & KURTA \\
\hline Brucella & BRCL & BRUC & BRUCL & Kusnezovia & KNZO & KUSZ & KUSNZ \\
\hline Butyrivibrio & BUVB & BUVB & BL & Lachnospira & LCSP & LACS & LACSP \\
\hline Calymmatobacterium & CMBT & LY & $\mathrm{CA}$ & Lactobacillus & LCBC & LACT & LACTB \\
\hline Campylobacter & CPBT & CAMP & CAMBC & Lamprocystis & LMCS & LAMC & LAMCS \\
\hline Cardiobacterium & CDBT & CARD & $\mathrm{CA}$ & Lampropedia & LMPD & LAMP & LAMPD \\
\hline Caryophanon & $R Y$ & & & spira & LPSP & LESP & LEPSP \\
\hline Caulobacter & CLBT & UB & & hrix & LPTX & LETH & LEPTX \\
\hline Caulococcus & CLCO & CAUC & CAUCO & ichia & LPTC & LETT & LETTC \\
\hline Cellulomonas & CEMN & CELM & & Leuconostoc & LEUC & LEUC & LEUCN \\
\hline Chlamydia & CLMD & CHAM & $\mathrm{CH}$ & othrix & LETX & LEUX & LEUTX \\
\hline Chlorobium & CLOR & CHLB & CHLBM & keella & LSKL & LISK & LISKL \\
\hline Chloropseudomonas & CSMN & CHLP & CHLMN & Listeria & LIST & LSTR & LSTRA \\
\hline Chondromyces & CNMY & CHNM & & Lucibacterium & LUBT & LUBC & LUCBC \\
\hline Chromatium & CMTM & CHRA & CHRAT & Macromonas & MAMN & MAMN & MACMN \\
\hline Chromobacterium & CRBT & CHRB & & Megasphaera & MGSF & MASF & MEGSF \\
\hline Citrobacter & CTBT & CITB & & Melittangium & MLNG & MEIT & MEITN \\
\hline Clathrochloris & CLCH & CLCH & CI & Metallogenium & MLOG & MELG & MELGN \\
\hline Clonothrix & CNTX & CLNT & CLNTX & Methanobacteriu & MEBT & MENB & MENBC \\
\hline Clostridium & CLOS & CLOS & CLOST & Methanococcus & MECO & MENC & MENCO \\
\hline Corynebacterium & CNBT & CNBC & $\mathrm{CON}$ & Methanosarcina & MESR & MENS & MENSA \\
\hline Cowdria & CWDR & COWD & Cow & Methylococcus & MLCO & MEYC & MEYCO \\
\hline Coxiella & COXL & COXL & COXLA & Methylomonas & MLMN & MEYM & MEYMN \\
\hline Crenothrix & CRTX & CRNX & CRNTX & Microbispora & MBSO & MICB & MICBS \\
\hline
\end{tabular}


TABLE 1-Continued

\begin{tabular}{|c|c|c|c|c|c|c|c|}
\hline Genus & $\begin{array}{c}\text { Johnson } \\
\text { et al. } \\
\text { proposal } \\
(1)\end{array}$ & $\begin{array}{c}\text { 4-Char- } \\
\text { acter al- } \\
\text { pha- } \\
\text { betic } \\
\text { pro- } \\
\text { posal }\end{array}$ & $\begin{array}{l}\text { 5-Charac- } \\
\text { ter alpha- } \\
\text { betic pro- } \\
\text { posal }\end{array}$ & Genus & $\begin{array}{l}\text { Johnson } \\
\text { et al. } \\
\text { proposal } \\
(1)\end{array}$ & $\begin{array}{c}\text { 4-Char- } \\
\text { acter al- } \\
\text { pha- } \\
\text { betic } \\
\text { pro- } \\
\text { posal }\end{array}$ & $\begin{array}{l}\text { 5-Charac- } \\
\text { ter alpha- } \\
\text { betic pro- } \\
\text { posal }\end{array}$ \\
\hline Micrococcus & MICO & MICO & MICCO & Sáprospira & SASP & SAPR & SAPRS \\
\hline Microcyclus & MICY & MICY & MICCY & Sarcina & SR & SARC & SARCN \\
\hline Microellobosporia & MLSO & MILS & MILSO & Selenomonas & SEMN & SELN & SELMN \\
\hline Micromonospora & MOSO & MIMS & MIMSO & Seliberia & SEBR & SELR & SELRA \\
\hline Micropolyspora & MPSO & MIPS & MIPSO & Serratia & SER & SERA & SERTA \\
\hline Moraxella & MRXL & MRXL & MRXLA & Shigella & SHGL & SHGL & SHIGL \\
\hline Mycobacterium & MYBT & MYBC & MYCBC & Siderocapsa & SDCP & SICA & SIDCA \\
\hline Mycoplasma & MYPL & MYPL & MYCPL & Siderococcus & SDCO & SICO & SIDCO \\
\hline Myхососсиs & MXCO & MYXC & MYXCO & Simonsiella & SMSL & SIMS & SIMSL \\
\hline Nannocystis & NACS & NACS & NANCS & Sphaerotilus & SFTL & SPAR & SPART \\
\hline Naumanniella & NMNL & NAUM & NAUML & Spirillospora & SPSO & SPLS & SPLSO \\
\hline Neisseria & NEIS & NEIS & NEISA & Spirillum & SP & SPLU & SPLUM \\
\hline Neorickettsia & NERK & NERK & NERKT & Spirochaeta & SPCT & SPRC & SPRCT \\
\hline Neuskia & NVSK & NEVS & NVSK & Spiroplasma & SPPL & SPRP & SPRPL \\
\hline Nitrobacter & NIBT & NIBC & NITBC & Sporichthya & SOCH & SRCH & SRCHT \\
\hline Nitrococcus & NICO & NICO & NITCO & Sporocytophaga & SOCY & SRCY & SRCYT \\
\hline Nitrosococcus & NOCO & NOCO & NOSCO & Sporolactobacillus & SOLC & SRLB & SRLBA \\
\hline Nitrosolobus & NOLB & NOLB & NOSLB & Sporosarcina & SOSR & SRSC & SRSRC \\
\hline Nitrosomonas & NOMN & NOMN & NOSMN & Staphylococcus & SFCO & STAF & STAFC \\
\hline Nitrosospira & NOSP & NOSO & NOSSO & Stigmatella & SGML & STAT & STMTL \\
\hline Nitrospina & NISN & NOSP & NOSSP & Streptobacillus & STBC & STBA & STRBA \\
\hline Nocardia & NOC & NRDA & NRDIA & Streptococcus & STCO & STCO & STRCO \\
\hline Ochrobium & OCRB & OCHB & OSCBM & Streptomyces & STMY & STMY & STRMY \\
\hline Oscillospira & OSSP & OSSP & OSCSP & Streptosporangium & STSO & STSO & STRSO \\
\hline Paracoccus & PRCO & PACO & PARCO & Streptothrix & STTX & STTX & STRTX \\
\hline Paranaplasma & PRPL & PAPL & PARPL & Streptoverticillium & STVC & STVC & STRVC \\
\hline Pasteurella & PSTL & PASL & PASTL & Succinimonas & SCMN & SUCM & SUCMN \\
\hline Pasteuria & PSIA & PAST & PASTR & Succinivibro & SCVB & SUCV & SUCVB \\
\hline Pediococcus & PDCO & PDCO & PEDCO & Sulfolobus & SULB & SULB & SULFB \\
\hline Pedomicrobium & PDMI & PDMI & PEDMI & Symbiotes & SMBI & SYMB & SYMBI \\
\hline Pelodictyon & PEDT & PEDT & PELDT & Thermoactinomyces & TMAT & TEAM & TEAMY \\
\hline Pelonema & PENM & PENM & PELNM & Thermomonospora & TMMO & TEMO & TEMNS \\
\hline Peloploca & PEPC & PEPC & PELPC & Thermoplasma & TMPL & TEPL & TEMPL \\
\hline Pelosigma & PESM & PESM & PELSM & Thermus & TM & TERM & TERMS \\
\hline Peptococcus & PTCO & PFiTC & PETCO & Thiobacillus & THBC & THBA & THIBA \\
\hline Peptostreptococcus & PSCO & PiTS & PETSC & Thiobacterium & THBT & THBC & THIBC \\
\hline Pfeifferella & Not listed & FFRL & PFERL & Thiocapsa & THCP & THCP & THICP \\
\hline Photobacterium & PHBT & P.HBC & PHOBC & Thiocystis & THCS & THCS & THICS \\
\hline Phragmidiothrix & PHTX & PHTX & PHRTX & Thiodendron & THDD & THDD & THIDD \\
\hline Pilimelia & PIML & PIML & PILML & Thiodictyon & THDT & THDT & THIDT \\
\hline Planctomyces & PTMY & PLCM & PLCMY & Thiopedia & THPD & THPD & THIPD \\
\hline Planobispora & PBSO & PLOB & PLOBS & Thioploca & THPC & THPL & THIPL \\
\hline Planococcus & PNCO & PLOC & PLOCO & Thiosarcina & THSR & THSC & THISC \\
\hline Planomonospora & PMSO & PLOM & PLOMS & $T$ & THSP & THSP & THISP \\
\hline Plesiomonas & PLMN & PLSM & PLSMN & Thiospirillum & THSL & THSR & THISR \\
\hline Polyangium & PONG & PONG & PONGM & Thiothrix & THTX & THTX & THITX \\
\hline Promicromonospora & Not listed & PRAM & PRMSO & Thiovulum & THVL & THVL & THIVL \\
\hline Propionibacterium & PRBT & PRBC & PRPBC & Toxothrix & TOTX & TOTX & TOXTX \\
\hline Prosthecochloris & PCCL & PRSC & PRSCL & onema & TREP & TREP & TREPN \\
\hline Prosthecomicrobium & PCMI & PRSM & PRSMI & Ureaplasma & Not listed & URPL & UREPL \\
\hline Proteus & PROT & PRTS & PRTUS & Veillonella & VEIL & VEIL & VEILN \\
\hline Pseudomonas & PSMN & PSMN & PSDMN & Vibrio & VB & VIBR & VIBRO \\
\hline Pseudonocardia & PSNC & PSNC & PSDNC & Vitreoscilla & VITR & VITR & VITRS \\
\hline Rhizobium & RHIZ & RHIZ & RHIZB & Wolbachia & WLBC & WLBC & WOLBC \\
\hline Rhodomicrobium & ROMI & RHMI & RHOMI & Xanthomonas & XNMN & XNMN & XANMN \\
\hline Rhodopseudomonas & ROPS & RHPS & RHOPS & Yersinia & YERS & YERS & YERSN \\
\hline Rhodospirillum & ROSP & RHSP & RHOSP & Zoogloea & ZOGL & ZOGL & ZOGLO \\
\hline Rickettsia & RKTS & RICK & RICKT & Zymomonas & ZYMN & ZYMN & ZYMMN \\
\hline Rickettsiella & RKSL & RISL & RIKSL & \multicolumn{4}{|c|}{ Genera of uncertain standing } \\
\hline Rochalimaea & RKLM & ROLM & ROCLM & Achromobacter & ACHR & ACHO & ACHRM \\
\hline Rothia & ROTH & ROTH & ROTHA & Actinomonospora & ATMO & ACTM & ACTMS \\
\hline Ruminococcus & RMCO & RUCO & RUMCO & Actinopycnidium & ATPN & ACTQ & ACTPY \\
\hline $\begin{array}{l}\text { Saccharomonospora } \\
\text { Salmonella }\end{array}$ & $\begin{array}{l}\text { Not listed } \\
\text { SAML }\end{array}$ & $\begin{array}{l}\text { SACS } \\
\text { SALM }\end{array}$ & $\begin{array}{l}\text { SACSO } \\
\text { SALMN }\end{array}$ & Actinosclerotium & ATSC & ACTR & ACTSC \\
\hline & & & & Actinosporangium & ATSO & ACTS & ACTSO \\
\hline
\end{tabular}


TABLE 1-Continued

\begin{tabular}{|c|c|c|c|}
\hline Genus & $\begin{array}{l}\text { Johnson } \\
\text { et al. } \\
\text { proposal } \\
\text { (1) }\end{array}$ & \begin{tabular}{|c|} 
4-Char- \\
acter al- \\
pha- \\
betic \\
pro- \\
posal
\end{tabular} & $\begin{array}{l}\text { 5-Charac- } \\
\text { ter alpha- } \\
\text { betic pro- } \\
\text { posal }\end{array}$ \\
\hline Ampullaria & APUL & * & * \\
\hline Aquaspirillum & AQSP & AQSP & AQASP \\
\hline Beneckea & SNKA & BENK & BENKA \\
\hline Betabacterium & BEBT & BETB & BETBC \\
\hline Betacoccus & BECO & BETC & BETCO \\
\hline Blastocaulis & BSCL & BLSC & BLSCA \\
\hline Brevibacterium & BVBT & BREV & BREVB \\
\hline Catenabacterium & CABT & CATB & CATBC \\
\hline Cellvibrio & CEVB & CELV & CELVB \\
\hline Chlorochromatium & CLMT & CHLC & CHLCM \\
\hline Cillobacterium & CIBT & CILB & CILBC \\
\hline Cladothrix & CLTX & CLAD & CLADX \\
\hline Diplococcus & DPCO & DIPC & DIPCO \\
\hline Eikenella & EKNL & EKNL & EKNLA \\
\hline Elytrosporangium & ELSO & ELSO & ELYSO \\
\hline Ferribacterium & FRBT & FERB & FERBC \\
\hline Fusiformis & FSFM & FSIF & FSIFM \\
\hline Fusocillus & FSCL & FSOC & FSOCL \\
\hline Gaffkya & GFKY & $* *$ & ** \\
\hline Herellea & HERL & $* *$ & ** \\
\hline Hydrogenomonas & HYMN & HYDM & HYDMN \\
\hline Intrasporangium & ITSO & ITSO & ITRSO \\
\hline Levinea & LEVN & LEVN & LEVNA \\
\hline Lingelsheimia & LNGM & LNGM & LNGSM \\
\hline Methanospirillum & MESP & MENU & MENSP \\
\hline Methylovibrio & MLVB & MEYV & MEYVB \\
\hline Microbacterium & MIBT & MIBA & MICBA \\
\hline Microtetraspora & MTSO & MITS & MITSO \\
\hline Mima & MIMA & $* *$ & $* *$ \\
\hline Myxobacter & MXBT & MYXB & MYXBC \\
\hline Oceanomonas & OCMN & OCMN & OCEMN \\
\hline Oceanospirillum & OCSP & OCNS & OCENS \\
\hline Odontomyces & ODMY & ODMY & ODTMY \\
\hline Oscillaria & OSLR & * & $*$ \\
\hline Oscillatoria & OSLT & * & * \\
\hline Pectobacterium & PCBT & PCBC & PCTBC \\
\hline Providencia & PRVD & PRVD & PRVDN \\
\hline Pseudobacterium & PSBT & PSBC & PSDBC \\
\hline Ramibacterium & RMBT & $\mathrm{RABC}$ & RAMBC \\
\hline Rhodothece & ROTC & RHTC & RHTCE \\
\hline Ristella & RSTL & RIST & RISTL \\
\hline Saccharomonas & SAMN & SACM & SACMN \\
\hline Siderobacter & SDBT & SIBC & SIDBC \\
\hline Sideromonas & SDMN & SIDM & SIDMN \\
\hline Sideronema & SDNM & SIDN & SIDNM \\
\hline Siderophacus & SDFC & SIDP & SIDPC \\
\hline Siderosphaera & SDSF & SIDS & SIDSF \\
\hline Sorangium & SRNG & SONG & SORNG \\
\hline Sphaerophorus & SFFR & SPAF & SPAFR \\
\hline Spironema & SPNM & SPRN & SPRNM \\
\hline Spiroschaudinnia & SPSD & SPSD & SPSCD \\
\hline Stelangium & SLNG & STAG & STANG \\
\hline Streptobacterium & STBT & STBC & STRBC \\
\hline Streptopycnidium & STPN & STPN & STRPN \\
\hline Sulfomonas & SUMN & SUMN & SULMN \\
\hline Sulfospirillum & SUSP & SUSP & SULSP \\
\hline Thermobacterium & TMBT & TEBC & TEMBC \\
\hline Thermopolyspora & TMPO & TEPO & TEMPO \\
\hline Thermostreptomyces & TMSM & TEPT & TEMPT \\
\hline Vertillomyces & VTMY & VEMY & VERMY \\
\hline Waksmania & WKMA & WKMA & WAKMA \\
\hline Zymobacterium & ZYBT & ZYBC & ZYMBC \\
\hline Zymosarcina & ZYSR & ZYSR & ZYMSR \\
\hline
\end{tabular}

* Not bacterial genera.

** Nomina rejicienda. by increasing the length of the abbreviation from four characters to five characters. To those not familiar with computers this is not necessarily as serious as may initially appear. Firstly, the number of characters has still been reduced from a maximum of 19 (STREPTOVERTICILLIUM) to 5 (almost a $75 \%$ reduction). Secondly, many computers (such as the PDP-10) store at least five characters in each "word" of memory (if only four characters are inserted in a word the remaining character positions are set as blanks) and comparisons are performed on a word-by-word basis. Therefore, these computers would take exactly the same time to compare four characters as they would to compare five characters.

An alphabetic five-character list is also specified (Table 1). This (i) has removed many of the inconsistencies of the alphabetic four-character list, (ii) corresponds more closely with the Code

TABLE 2. Amended code for the abbreviations of names of bacterial genera appearing in the eighth edition of Bergey's Manual

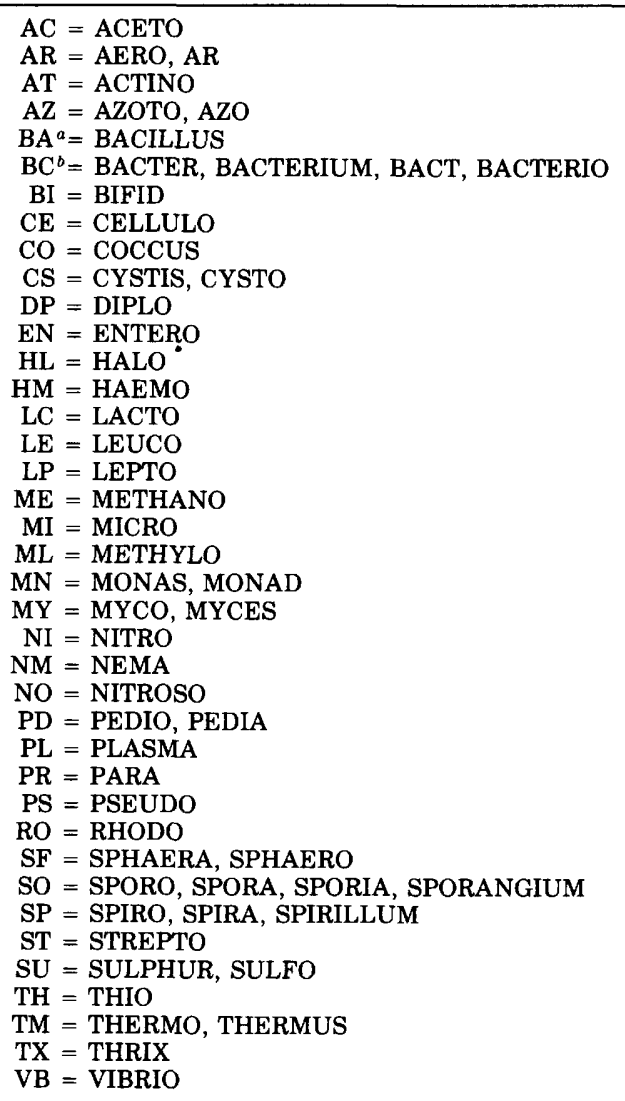

${ }^{a}$ Changed from $\mathrm{BC}=\mathrm{BACILLUS}$ in Johnson et al. (1).

${ }^{\circ}$ Changed from BT=BACTER, BACTERIUM, BACT, BACTERIO in Johnson et al. (1). 
for Abbreviations (Table 2), (iii) provides better correspondence between the generic name and abbreviation which is of importance for performing "human" conversions.

Johnson et al. specify an upper limit of four characters for each abbreviation; some have two or three characters. The proposed four- and five-character lists do not allow such variation. In many cases computer programming becomes much simpler if it is known that all abbreviations are always a fixed length (either four or five, whichever proposal is adopted).

\section{APPENDIX}

FORTRAN listing of subroutine to perform binary search by computer

SUBROUTINE SRCH (LOC, ARRAY, DIMEN, INDEX, \$)

C THIS SUBROUTINE SEARCHES TABLE 'ARRAY' OF LENGTH 'DIMEN' FOR THE VALUE CONTAINED IN 'LOC' ROW NUMBER IN TABLE

C IF NOT FOUND, THE \$ RETURN IS TAKEN
INTEGER LOC, INDEX, P, Q, DIMEN, ARRAY (DIMEN)

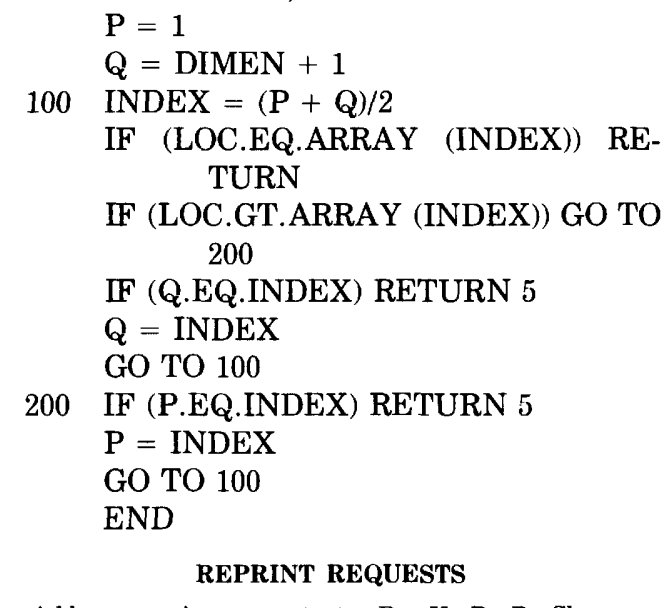

Address reprint requests to: Dr. V. B. D. Skerman, World Data Center for Microorganisms, Department of Microbiology, University of Queensland, St. Lucia, Queensland, Australia 4067.

\section{LITERATURE CITED}

1. Johnson, R., M. Rogosa, and M. I. Krichevsky. 1976. Abbreviations of names of genera suggested for coding microbiological data. Int. J. Syst. Bacteriol. 26:278-282. 